\title{
MicroRNA-128 promotes cell-cell adhesion in U87 glioma cells via regulation of EphB2
}

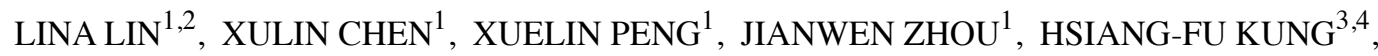 \\ MARIE C. LIN ${ }^{5}$ and SONGSHAN JIANG ${ }^{1}$
}

\begin{abstract}
${ }^{1}$ Key Laboratory of Gene Engineering of the Ministry of Education, State Key Laboratory of Biocontrol, School of Life Sciences, Sun Yat-sen University, Guangzhou; ${ }^{2}$ Center for Reproductive Medicine, Affiliated Shantou Hospital of Sun Yat-sen University, Shantou; ${ }^{3}$ School of Biomedical Science and Li Ka Shing Institute of Health Sciences, The Chinese University of Hong Kong, Hong Kong SAR; ${ }^{4}$ State Key Laboratory of Oncology in Southern China, Cancer Center,

Sun Yat-sen University, Guangzhou; ${ }^{5}$ Brain Tumor Centre, Division of Neurosurgery, Department of Surgery,

Faculty of Medicine, The Chinese University of Hong Kong, Shatin, Hong Kong SAR, P.R. China
\end{abstract}

Received April 9, 2013; Accepted June 3, 2013

DOI: $10.3892 / o r .2013 .2596$

\begin{abstract}
MicroRNAs (miRNAs) are small, non-coding RNAs which regulate gene expression at the post-transcriptional level. Abnormal expression of miRNAs occurs frequently in human tumors. Despite the fact that reduced expression of miR-128 has been observed in glioma tissues and cells, the role of miR-128 in tumors has not been fully characterized. In the present study, cell adhesion assays indicated that overexpression of miR-128 can promote cell-cell adhesion. Target site prediction algorithms indicated that miR-128 binds the 3'-untranslated regions of erythropoietin-producing hepatocellular receptor (Eph)B1 and EphB2 mRNAs. Luciferase reporter assays confirmed that miR-128 binds and regulates EphB1 and EphB2 mRNAs. Overexpression of EphB2 reduced the ability of miR-128 to promote cell-cell adhesion. The wound-healing assay indicated that miR-128 significantly inhibited cell migration via EphB2. This study revealed the novel functions of miR-128 in cell-cell adhesion and cell migration in glioma cells through the regulation of EphB2, and identified EphB1 and EphB2 as novel miR-128 targets.
\end{abstract}

\section{Introduction}

Gliomas are the most common primary tumors in the central nervous system. Glioblastoma multiforme (GBM, grade IV

Correspondence to: Professor Songshan Jiang, Key Laboratory of Gene Engineering of the Ministry of Education, State Key Laboratory of Biocontrol, School of Life Sciences, Sun Yat-sen University, 132 East Waihuan Road, Room 312, Higher Education Mega, Guangzhou, Guangdong 510006, P.R. China

E-mail: jiangssh@mail.sysu.edu.cn

Key words: microRNA-128, glioma, cell-cell adhesion, erythropoietinproducing hepatocellular receptor B2 according to WHO classification) is one of the most lethal human tumors, accounting for almost half of malignant brain tumors. Glioblastoma is highly invasive and usually recurs even after aggressive resection, radiation or chemotherapy (1). Since the prognosis for patients with high-grade glioma, including anaplastic astrocytoma and GBM, remains dismal, novel therapeutic approaches are required.

MicroRNAs (miRNAs) are single stranded 21-25 nucleotide non-coding RNAs, which post-transcriptionally regulate the expression of protein-coding genes through perfect or imperfect base pairing with the 3'-untranslated region (3'UTR) of target mRNAs (2). The role of miRNAs in tumors has been studied extensively and they are considered to be novel therapeutic targets. miRNAs are frequently located in genomic regions which are amplified, deleted or suffer loss of heterozygosity in cancer (3) and miRNAs can regulate the expression of tumor-associated genes in multiple tumor types, including gliomas (4-7).

The brain-enriched miR-128 is an important miRNA in several tumors, with a varied expression pattern (8). miR-128 is strongly downregulated in gliomas, compared to normal brain tissues $(6,9)$. Previous studies have shown that miR-128 is involved in cell death/survival processes via targeting the truncated isoform of NTRK3 in SH-SY5Y neuroblastoma cells (10) and the Bax protein in human embryonic kidney cells (11); it can inhibit glioma cell proliferation via downregulation of the transcription factor E2F3a in glioma cells $(12,13)$, it can reduce glioma self-renewal by inhibition of Bmi-1 expression (13) and it can also participate in neuroblastoma cell motility and invasiveness by inhibiting Reelin and DCX (14).

The erythropoietin-producing hepatocellular receptors (Eph) are the largest sub-family of receptor tyrosine kinases (RTKs), and their ligands, the ephrins, compose a major repulsive signaling system. The erythropoietin-producing hepatocellular receptor B2 (EphB2), a member of the Eph receptor tyrosine kinase family, has been shown to regulate cytoskeleton organization and cell migration in various cell 
types (15). The EphB2 expression has been shown to be upregulated in gliomas as compared to normal brain tissues (16), and overexpression of EphB2 reduces cell adhesion and increases cell invasion in glioma tissues and cells $(16,17)$.

In this study, we report a novel function of miR-128 in glioma cells. We have demonstrated that overexpression of miR-128 in glioma cells increased cell-cell adhesion and inhibited cell migration. Furthermore, we have identified the erythropoietin-producing hepatocellular receptor B1 (EphB1) and EphB2 as novel targets of miR-128, and have shown that miR-128 promotes cell-cell adhesion by regulating the EphB2. The results of this study provide new insight into the mechanism by which miR-128 inhibits glioma cell migration into, and invasion of, the surrounding tissues.

\section{Materials and methods}

Cell culture. The human U87 glioma cell line and HEK-293T (293T) cell line were purchased from the American Type Culture Collection (ATCC, Gaithersburg, MD, USA). Both cell lines were cultured in Dulbecco's Modified Eagle's Medium (DMEM)/High Glucose medium (Thermo Scientific) supplemented with $10 \%$ fetal bovine serum (FBS) and $100 \mathrm{U} / \mathrm{ml}$ penicillin/streptomycin.

Vector construction. For expression of miR-128, a $287 \mathrm{bp}$ genomic fragment containing the human miR-128-2 precursor and flanking sequences was amplified using the primers listed in Table I and cloned into the modified pll3.7 vector under the control of the human U6 promoter. This construct was termed pmiR-128. To construct luciferase reporter vectors, the 3'UTR fragments containing the putative miR-128 binding sequences of human EphB1 (655 bp) and EphB2 (856 bp) were amplified and cloned downstream of Renilla luciferase in the modified psiCheck 2 vector. For overexpression of EphB2, the full length EphB2 coding sequence without the 3'UTR was amplified from U87 cell total RNA by RT-PCR and cloned into pcDNA3.1 (Invitrogen).

The mutant luciferase reporter constructs psiCheck2EphB1-3'UTR-MUT or psiCheck2-EphB2-3'UTR-MUT carrying mutations in the sequence of the complementary seed region miR-128 site were generated by fusion PCR. The 655 and 856 bp amplified 3'UTRs were divided into two fragments in which the mutated sequence was introduced into the overlapping regions. Then, the two purified PCR products were mixed and amplified using primers for the whole 655 or 856 bp 3'UTR fragment. All the primers used are listed in Table I.

Lentivirus production and establishment of stable cell lines. VSV-G pseudotyped lentiviruses were produced by co-transfection of $293 \mathrm{~T}$ cells with the transfer vector and three packaging vectors: pMDLg/pRRE, pRSV-REV and pCMV-VSVG. Then, the production medium containing lentivirus was harvested, centrifuged to remove cell debris and viral supernatant was used for infection.

U87 cells were seeded at $30 \%$ confluence in a $25 \mathrm{~cm}^{2}$ dish in preparation for lentiviral infection and $24 \mathrm{~h}$ later, $8 \mu \mathrm{g} / \mathrm{ml}$ polybrene (Sigma) was added to the media, the cells were infected with lentivirus and $48 \mathrm{~h}$ later $0.25 \mu \mathrm{g} / \mathrm{ml}$ puromycin
(Enzo Life Sciences, Farmingdale, USA) was added to the culture media to select U87 infected cells.

RNA extraction and quantitative reverse-transcription $P C R$ ( $q R T-P C R)$. Total RNA was extracted using TRIzol(Invitrogen) and quantification of mature miRNA using qRT-PCR was performed using primers listed in Table I. Briefly, total RNA was used to synthesize cDNA with the Reverse Transcriptase (RT)-PCR kit (Toyobo, Japan) and a stem-loop-like RT primer containing a miRNA specific region in the $3^{\prime}$ end (Gene Science and Health, China). qRT-PCR was performed using the StepOne ${ }^{\mathrm{TM}}$ Real-Time PCR system (Applied Biosystems) and the miRNA qPCR Quantitation kit (Gene Science and Health) according to the manufacturer's instructions. The qRT-PCR cycle conditions were: denaturation at $95^{\circ} \mathrm{C}$ for $10 \mathrm{~min}$, followed by 40 cycles of $95^{\circ} \mathrm{C}$ for $15 \mathrm{sec}$ and $60^{\circ} \mathrm{C}$ for $1 \mathrm{~min}$. Each sample, including the no template control, was run in triplicate and $C_{t}$ values were determined for the target transcripts using fixed threshold settings. Cellular miRNA expression was normalized to U6 snRNA.

Cell-cell adhesion assay. Cells were plated in DMEM containing $10 \%$ FBS. Images of cell-cell contact sites were captured under an inverted fluorescence microscope (DIC optics; model TE2000; Nikon, Japan) with a x40 air Fluor objective using a 12-bit CCD camera and Image Pro software (Nikon). The frequency of adhesions between cells expressing miR-128, shRNA2 or control cells was analyzed. Cell-cell adhesion was defined as a cell-cell contact area of $>50 \%$. At least 100 contact sites were randomly selected, and three independent replicates were performed.

Dual-luciferase reporter assay. The dual-luciferase reporter assay was carried out as previously described (18). Briefly, $2.5 \times 10^{4} 293 \mathrm{~T}$ cells in $100 \mu \mathrm{l}$ growth medium were plated in 96-well plates, $2 \times 10^{4}$ U87 cells in $200 \mu 1$ growth medium were plated in 48 -well plates. The next day, the cells were transfected with $100 \mathrm{ng}$ psiCheck2-EphB1-3'UTR, psiCheck2EphB2-3'UTR or psiCheck2-EphB2-3'UTR-MUT and $300 \mathrm{ng}$ pmiR-128 or pmiR-CTRL using Lipofectamine ${ }^{\mathrm{TM}} 2000$ (Invitrogen) or FuGENE (Roche Applied Science). The cells were harvested $48 \mathrm{~h}$ after transfection and assayed using the Dual-Luciferase Reporter Assay kit (Promega) according to the manufacturer's instructions. Each transfection was repeated in triplicate.

Immunoblotting analysis. Cells were solubilized in lysis buffer at $100^{\circ} \mathrm{C}$ for $30 \mathrm{~min}$ and centrifuged at $12,000 \mathrm{x} \mathrm{g}$ for $10 \mathrm{~min}$ at $4^{\circ} \mathrm{C}$ to obtain whole-cell protein extract supernatants. The protein samples were resolved by SDS-polyacrylamide gel electrophoresis (PAGE), transferred to Immobilon-P membranes (Millipore), incubated in TBS containing 0.2\% Tween-20 and $5 \%$ skimmed milk to block non-specific binding overnight at $4^{\circ} \mathrm{C}$, then incubated for $2 \mathrm{~h}$ at room temperature with a primary rabbit polyclonal antibody against human EphB2 $(0.2 \mu \mathrm{g} / \mathrm{ml}$ dilution; R\&D Systems) or mouse monoclonal antibody against human $\beta$-actin (1:2,000 dilution; Sigma). The membranes were washed three times for $10 \mathrm{~min}$ in TBS containing $0.2 \%$ Tween-20, incubated for $1 \mathrm{~h}$ with goat anti-rabbit $\operatorname{IgG}(\mathrm{H}+\mathrm{L})$ (1:30,000; Jackson ImmunoResearch) or goat anti-mouse IgG 
Table I. Primers used for vector construction and quantitative Reverse Transcription PCR (qRT-PCR).

\begin{tabular}{ll}
\hline Gene & \\
\hline miR-128 precursor & F: CACAAGTCr $\left(5^{\prime} \rightarrow 3^{\prime}\right)$ \\
& R: AAGGATCCTTCCCATTACTAATTCTGCTTC \\
EphB1-3'UTR & F: CACAACTCGAGAACTCTTGTTTCTTGGGGAAGGAG \\
& R: AAAGATCTATGCAAACAAAAGAAAAACGAGGT \\
EphB2-3'UTR & F: CACAACTCGAGGATCCTGCATCTGGGTTTGTTTAC \\
& R: AAGGATCCTTCACTAACTGATTGCTCTGCTTG \\
EphB1-3'UTR-MUT & F: GAGGGAAAAGGACCAGGGTCATGGCGCTGGAGACCAAGACGTGACACGAATGTACTG \\
& R: CCAGTCTCTCCAGTACATTCGTGTCACGTCTTGGTCTCCAGCGCCATGACCCTGGTC \\
EphB2-3'UTR-MUT & F: GGCCAGGACCCGGATCAAAGTGACACTTACCCTGCCCTCCAGAGG \\
& R: CCTCTGGAGGGCAGGGTAAGTGTCACTTTGATCCGGGTCCTGGCCT \\
EphB2 expression & F: CACAAGTCGACGAAGCGCAACCATGGCTCTGCGGAG \\
& R: AAGGATCCAGTGTCACTTTGATCGGGGACTCAAA \\
miR-128 & RT: CTCAACTGGTGTCGTGGAGTCGGCAATTCAGTTGAGAAAGAGAC \\
& F: CCAGCTGGGTCACAGTGAACCGGT
\end{tabular}

$\mathrm{F}$, forward primer; $\mathrm{R}$, reverse primer.

(H+L) (1:10,000 dilution; Jackson ImmunoResearch) secondary antibodies, washed thoroughly and the bound antibodies were detected using enhanced chemiluminescence.

Wound-healing assay. Cell culture conditions were optimized to ensure homogeneous and viable cell monolayers prior to wounding. When the cell confluence reached $\sim 90 \%$, a homogenous artificial wound was created using a sterile plastic $1,000 \mu \mathrm{l}$ micropipette tip and cell debris was removed by washing the cells in serum-free medium. Following incubation for 12 and $24 \mathrm{~h}$, cell migration into the wounded area was photographed using an inverted microscope (magnification, x100).

Cell migration assay. Cells $\left(5 \times 10^{4}\right)$ in $200 \mu 1$ DMEM without FBS were seeded on a polycarbonate membrane which was inserted in a transwell apparatus (Costar, Cambridge, MA, USA). DMEM $(600 \mu \mathrm{l})$ with $10 \%$ FBS was added as a chemoattractant in the lower chamber. The insert was washed with PBS after the cells were incubated for $4 \mathrm{~h}$ at $37^{\circ} \mathrm{C}$ in a $5 \% \mathrm{CO}_{2}$ atmosphere, and cells on the top surface of the insert were removed with a cotton swab. Cells adhering to the lower surface were fixed with $4 \%$ paraformaldehyde, stained with crystal violet solution and counted under a microscope in five random fields.

Statistical analysis. Data are presented as the means \pm SD of at least three separate experiments. All data are analyzed using the Student's t-test. Differences were considered statistically significant at $\mathrm{P}<0.05\left({ }^{*} \mathrm{P}<0.05 ;{ }^{* *} \mathrm{P}<0.01 ;{ }^{* * *} \mathrm{P}<0.001\right)$.

\section{Results}

miR-128 promotes cell-cell adhesion in U87 glioma cells. Expression of miR-128 is significantly decreased and negatively correlates with tumor grade in gliomas $(12,19)$. Functional studies indicate that miR-128 plays an important role in glioma tumorigenesis, including cell proliferation, self-renewal and invasion (12-14); however, the role of miR-128 in cell-cell adhesion and other functions has received little attention. In order to evaluate the role of miR-128 in glioma cell-cell adhesion, we constructed a U87 cell line overexpressing miR-128 (U87-miR-128 cells) using lentivirus infection and puromycin selection. qRT-PCR indicated a 2.3-fold increase in miR-128 expression in U87-miR-128 cells (Fig. 1A). U87-miR-128 cells clustered together more strongly in cell-cell adhesion assays (Fig. 1B and C) suggesting that miR-128 may promote cell-cell adhesion in U87 cells.

miR-128 regulates EphB2 expression through the 3'UTR binding site. miRNAs regulate downstream gene expression via binding to the 3'UTRs of target mRNAs. In order to find miR-128 target genes, we used the target prediction database miRanda (http://cbio.mskcc.org/mirnaviewer/) (20). A list of 744 putative target genes were generated and ranked by the binding energy, which estimates the thermodynamic properties of a predicted duplex and represents the likelihood of an interaction between a miRNA and mRNA. The top ten predicted targets, ranked according to the binding energy, are listed in Table II. Notably, two members of the Eph family, EphB1 and EphB2, were potential miR-128 targets. To improve the robustness of prediction, five other target prediction algorithms were used to predict the interaction between miR-128 and EphB1 and EphB2. EphB2 was predicted to be a mir-128 target gene by all five algorithms and EphB1 was predicted by two algorithms (Fig. 2A). In addition, no other Eph family members were predicted by any of the five algorithms. The Eph family is of particular interest as: i) previous studies have demonstrated that they are related to cell repulsion and adhesion (21-23); ii) the expression levels of some Eph family 
A

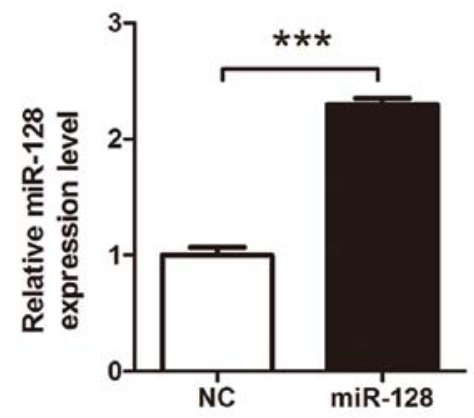

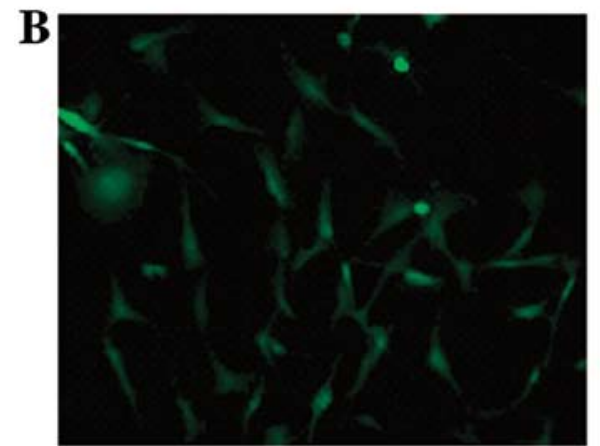

NC

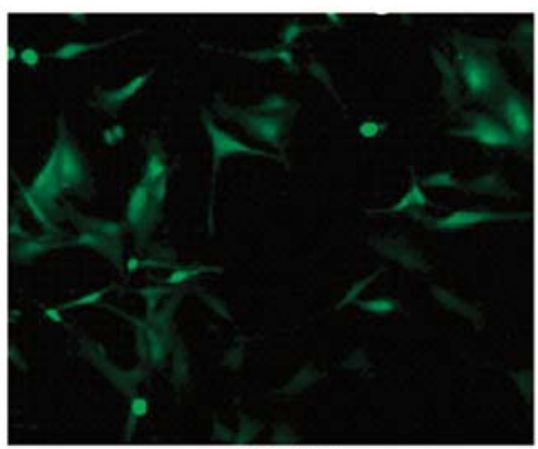

$\mathrm{miR}-128$

C

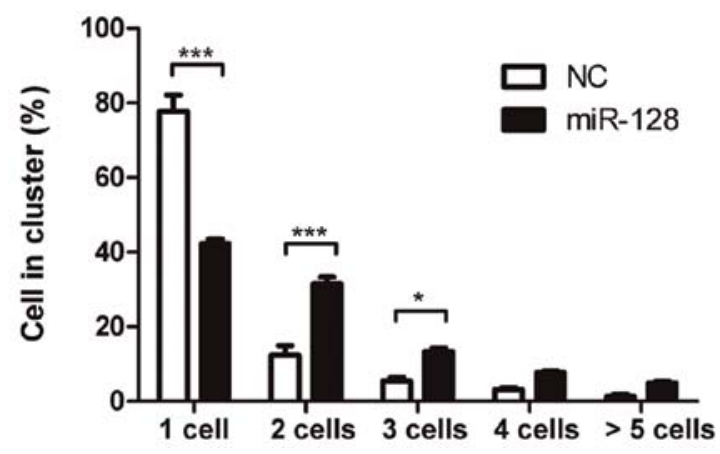

Figure 1. miR-128 promotes cell-cell adhesion in U87 glioma cells. (A) Quantitative real-time PCR quantification of miR-128 expression in stable miR-128-expressing U87 cells (miR-128) and control empty lentivirus-infected U87 cells [negative control (NC)]. (B) Representative fluorescence microscope image of stable miR-128-expressing U87 cells (miR-128) and control empty lentivirus-infected U87 cells (NC) $48 \mathrm{~h}$ after seeding. (C) Quantification of cell clustering in stable miR-128-expressing U87 cells (miR-128) and control empty lentivirus-infected U87 cells (NC). Cells were plated at moderate density, incubated for $48 \mathrm{~h}$ and the cell adhesion assay was performed. A total of 100 fields of view were randomly selected, and the number of clusters containing different number of cells was determined. Values are mean $\pm \mathrm{SD}(\mathrm{n}=3)$ of one representative experiment from several replicates. ${ }^{* * *} \mathrm{P}<0.001 ;{ }^{*} \mathrm{P}<0.05$.

members are increased in gliomas (12), in contrast to reduced miR-128 expression (6,9); iii) the EphB1 and EphB2 3'UTRs both contain a complementary site for the miR-128 seed region (2-8 nt) and are both predicted to form classic duplexes with miR-128 (Fig. 2B) (24); and iv) both the putative target sites are conservative, shown by 46 -way vertebrate alignments (Fig. 2B).

To confirm whether miR-128 binds directly to the 3'UTRs, we constructed two luciferase reporter vectors containing the predicted miR-128 binding site fragments from the human EphB1 and EphB2 3'UTRs. The vectors were co-transfected with miR-128 into $293 \mathrm{~T}$ cells. Ectopic expression of miR-128 reduced the activity of the luciferase reporter plasmids containing the EphB1 and EphB2 3'UTR miR-128 binding sites (Fig. 3A). Mutating the reporter vectors containing the EphB1 or EphB2 3'UTR miR-128-binding sites using a fusion PCR method and co-transfecting with miR-128, miR-128 had no effect on the luciferase activity of either mutant (Fig. 3B, C and G). The results of experiments were confirmed in U87 cells (Fig. 3D-F). These results suggest that miR-128 regulates luciferase activity via the predicted binding sites in the 3'UTRs EphB1 and EphB2 mRNAs. As EphB1 and EphB2 belong to the same Eph sub-family and miR-128 reduced the luciferase activity of the reporter vector containing the EphB2 3'UTR more significantly than EphB1, we selected EphB2 for further investigation.

To further determine the function of miR-128 as a regulator of EphB2, we investigated the effect of miR-128 on endogenous EphB2 expression. The qRT-PCR quantification of endogenous EphB2 mRNA indicated that ectopic stable expression of miR-128 in U87 glioma cells did not affect the mRNA level of EphB2 (Fig. 3H). However, immunoblotting showed that ectopic stable expression of miR-128 in U87 glioma cells reduces EphB2 expression at the protein level (Fig. 3I). Consistently, the increase of miR-128 mimics in U87 cells resulted in the decrease of EphB2 protein (Fig. 3J, left panel). Furthermore, introduction of miR-128 to U87 cells by transfecting miR-128 mimics and then increasing 
A

\begin{tabular}{|c|c|c|c|c|c|c|}
\hline & TargetScan & DIANA micro_T & PioTar & EIMMo & miRanda & microRNA.org \\
\hline EphB1 & & & & Yes & Yes & Yes \\
\hline EphB2 & Yes & Yes & Yes & Yes & Yes & Yes \\
\hline
\end{tabular}

B
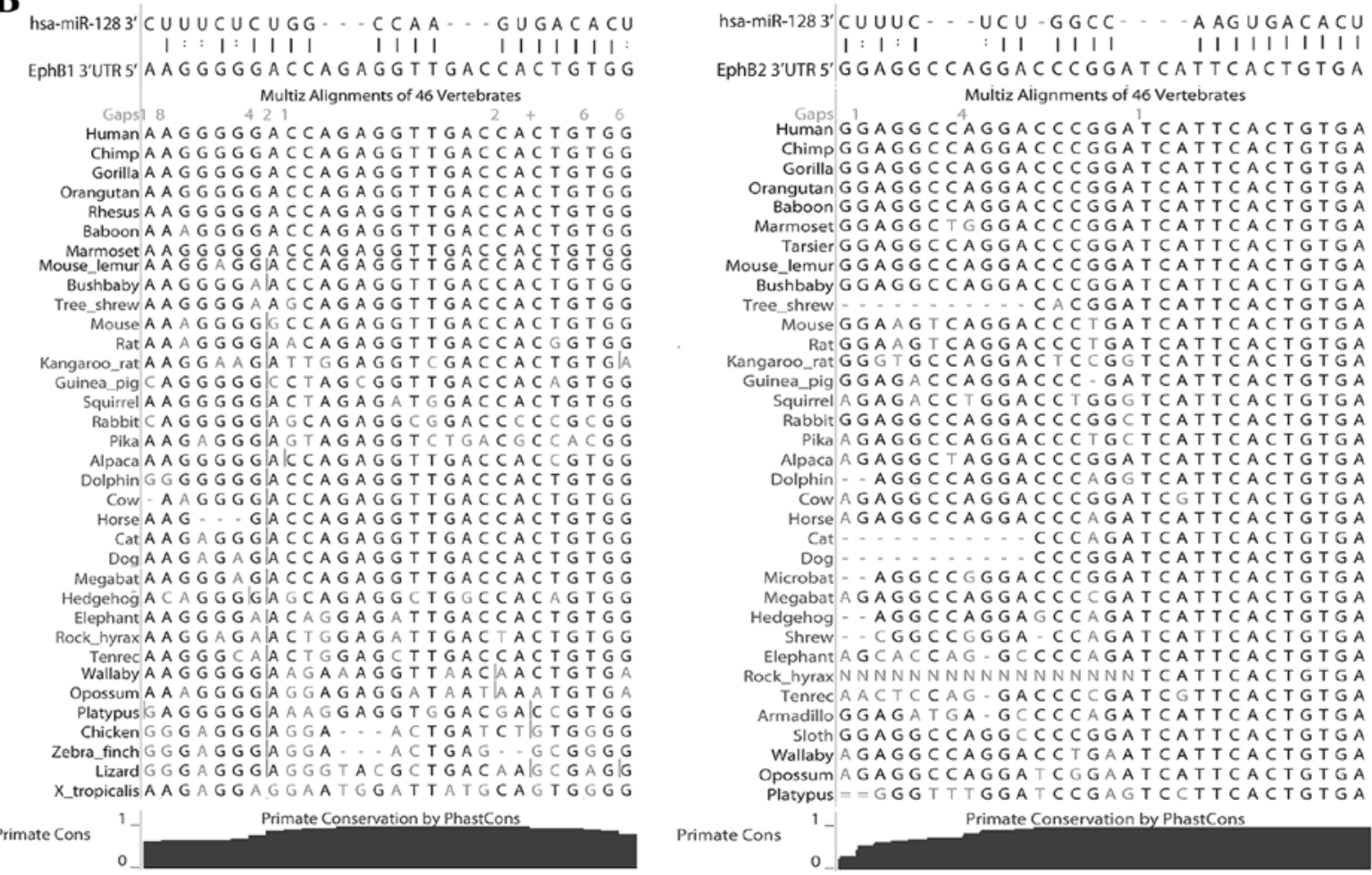

Figure 2. The erythropoietin-producing hepatocellular receptor B1 (EphB1) and the erythropoietin-producing hepatocellular receptor B2 (EphB2) are predicted miR-128 targets. (A) Interactions predicted between miR-128 and EphB1 and EphB2 by the target prediction algorithms TargetScan, DIANA micro_T, PicTar, EIMMo, miRanda, microRNA.org. EphB1 was predicted as a putative target of miR-128 by three algorithms (Yes) and EphB2 was predicted by all six algorithms. All programs predicted the same site for each gene. (B) Putative miR-128 target sites in the EphB1 and EphB2 3'-untranslated regions (3'UTRs) shown with the conservation tracks, including 46-way vertebrate alignments and phastCons-predicted conserved elements for primates.

Table II. The top 10 targets of miR-128 predicted by the miRanda algorithm.

\begin{tabular}{lccc}
\hline Gene & $\begin{array}{c}\text { Ensembl } \\
\text { gene ID }\end{array}$ & $\begin{array}{c}\text { Binding energy } \\
(\mathrm{kCal} / \mathrm{mol})\end{array}$ & $\begin{array}{c}\text { miRanda } \\
\text { score }\end{array}$ \\
\hline LRRC1 & ENSG00000137269 & -34.1 & 164 \\
KPNB1 & ENSG00000108424 & -30.5 & 152 \\
TMEM1 & ENSG00000160218 & -29.9 & 170 \\
HMGB3 & ENSG00000029993 & -29.5 & 165 \\
FRMD4 & ENSG00000151474 & -29.4 & 175 \\
TTN & ENSG00000155657 & -28.1 & 171 \\
EphB1 & ENSG00000154928 & -28.0 & 172 \\
EphB2 & ENSG00000133216 & -27.0 & 172 \\
SEC61A1 & ENSG00000058262 & -26.8 & 170 \\
SLC1A2 & ENSG00000110436 & -26.8 & 150 \\
\hline
\end{tabular}

All 744 predicted genes were ranked by the binding energy, and the top ten are presented.

miR-128 antisense RNA resulted in the increase of EphB2 protein (Fig. 3J, right panel). These results further indicated that miR-128 suppressed EphB2 expression via the miR-128binding site in the EphB2 mRNA 3'UTR.
To determine whether reduced EphB2 expression mimics the effect of miR-128 overexpression on cell-cell adhesion, two EphB2 shRNAs were constructed and transiently transfected into U87 cells. The expression level of endogenous EphB2 protein was inhibited more prominently by shRNA2 than shRNA1 (Fig. 4A), thus, shRNA2 was selected for further experiments. The cell-cell adhesion assay was performed in U87 glioma cells transfected with EphB2 shRNA2. EphB2 shRNA2-transfected cells displayed increased cell clustering compared to empty vector-transfected control cells. Notably, the effect of EphB2 knockdown on cell-cell adhesion was similar to miR-128 overexpression (Fig. 4B).

EphB2 suppresses miR-128-mediated increase of cell-cell adhesion. To further investigate the hypothesis that the phenotype resulting from miR-128 overexpression was due to downregulation of EphB2, miR-128-overexpressing U87-miR-128 cells were transfected with the EphB2 expressing vector $\mathrm{pEphB2}$. As the $\mathrm{pEphB} 2$ expression vector contains only the EphB2 coding sequences, but not the 3'UTR, miR-128 cannot affect the expression of EphB2 by pEphB2. Immunoblotting indicated that EphB2 expression increased in U87-miR-128 cells transfected with pEphB2, compared to the negative control-transfected U87-miR-128 cells or empty vector (Fig. 5A). Additionally, pEphB2 transfection reduced 

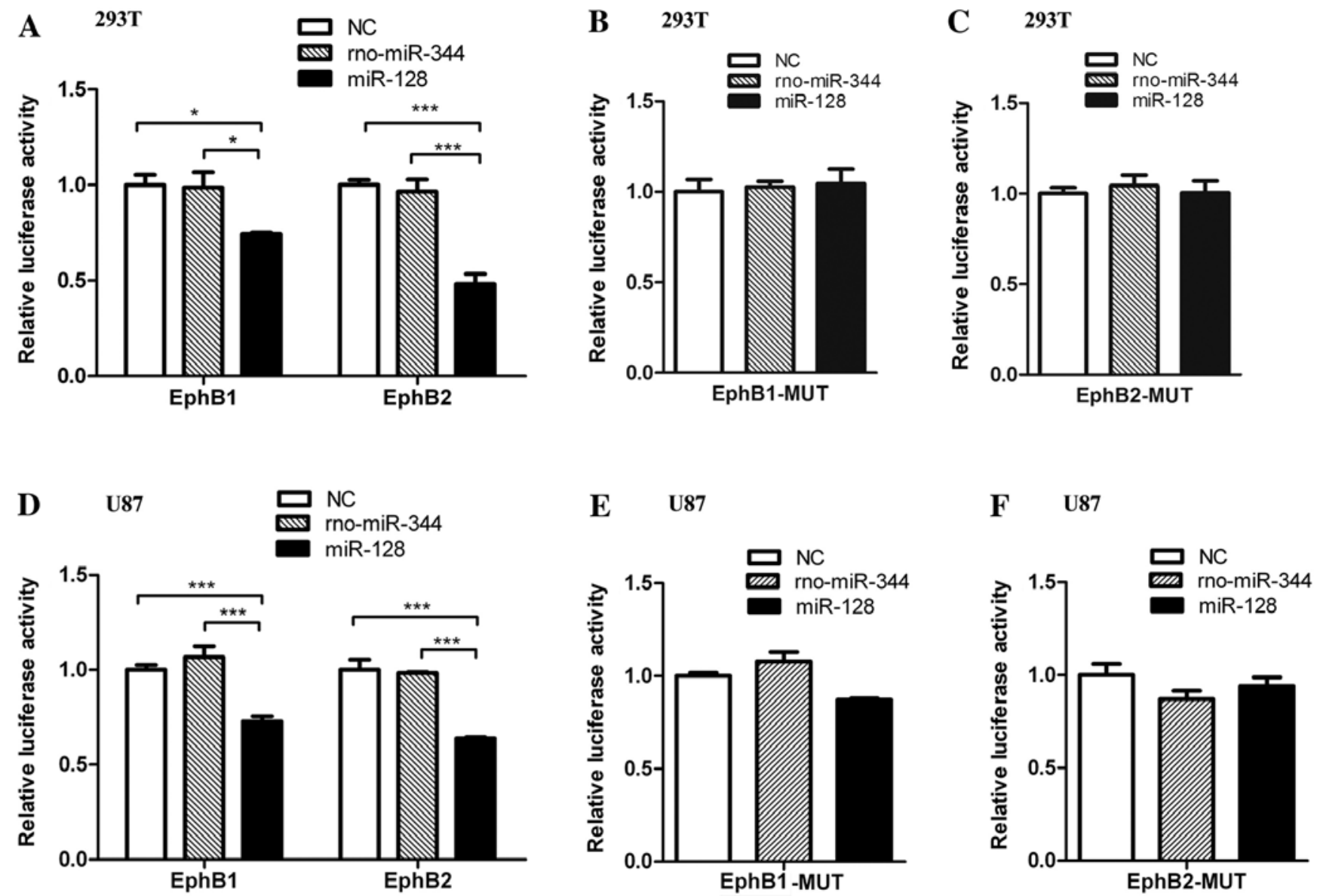

G

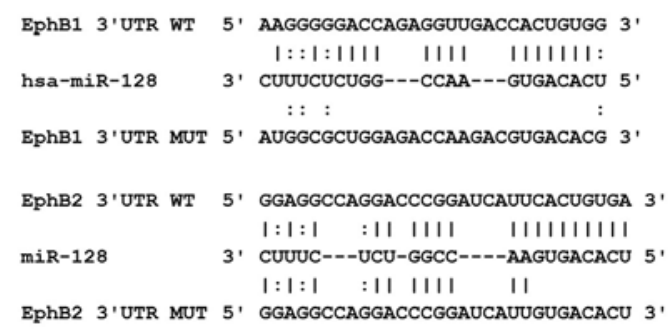

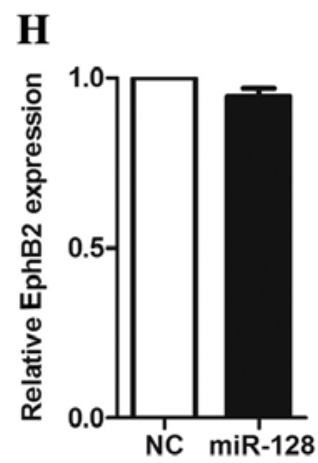

I

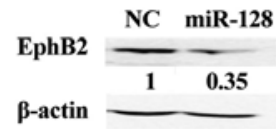

$\mathbf{J}$
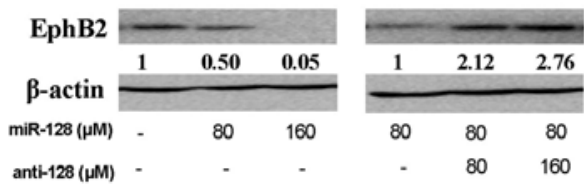

Figure 3. The erythropoietin-producing hepatocellular receptor B2 (EphB2) is a functional target of miR-128. (A) Luciferase reporter vectors psiCheck2EphB1-3'UTR (EphB1) or psiCheck2-EphB2-3'UTR (EphB2) containing the 3'UTR of EphB1 or EphB2 fused to Renilla luciferase were co-transfected into 293T cells with either a miR-128 expressing vector (miR-128) or empty vector [negative control (NC)] and luciferase activity was assayed $48 \mathrm{~h}$ later. The relative luciferase activities are the ratio of Renilla/Firefly luciferase normalized to the negative control (NC) cell activity. Values are mean \pm SD ( $\mathrm{n}=3$ ) of one representative experiment from several replicates; ${ }^{* * *} \mathrm{P}<0.001,{ }^{* *} \mathrm{P}<0.01$. (B and C) 293T cells were co-transfected with the (B) psiCheck2-EphB1-3'UTR-MUT (EphB1-MUT) or (C) psiCheck2-EphB2-3'UTR-MUT (EphB2-MUT) plasmids containing the mutated EphB1-3'UTR or mutated EphB2-3'UTR fused to Renilla luciferase and either a miR-128 expression vector (miR-128), the rat miR-344 expression vector (rno-344) or empty vector control (NC) and luciferase activity was assayed $48 \mathrm{~h}$ later. The relative luciferase activities are the ratio of Renilla/Firefly luciferase normalized to the negative control (NC). Values are means of at least three independent experiments performed in duplicate. (D-F) The same experiments of (A-C) were assayed within U87 cells. (G) The putative EphB1 and EphB2 3'UTR miR-128-binding sequences. Mutations were generated in the EphB1 and EphB2 3'UTR complementary seed region site or full miR-128 binding region, as indicated, to generate the EphB1-MUT and EphB2-MUT plasmids. (H-J) Expression of endogenous EphB2 is suppressed by miR-128. The qRT-PCR quantification of $(\mathrm{H})$ endogenous EphB2 mRNA levels and (I and J) immunoblotting of endogenous EphB2 protein levels in stable miR-128-expressing U87 cells (miR-128) compared with (I) empty vector control cells (NC), and (J) U87 cells transfected with increased miR-128 mimics or both miR-128 mimics and increased antisenses. $\beta$-actin was used as a loading control and normalized band densities are shown in (I andJ).

the number of clustering cells in the cell-cell adhesion assay, compared to controls of U87-miR-128 cells or U87 cells transfected with empty vector (Fig. 5B). These results indicate that overexpression of EphB2 can rescue the cell-cell adhesion effect induced by miR-128 overexpression, suggesting that EphB2 mediates miR-128-regulated cell-cell adhesion. 
A

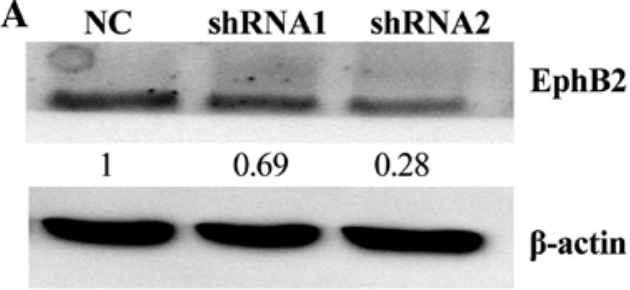

B

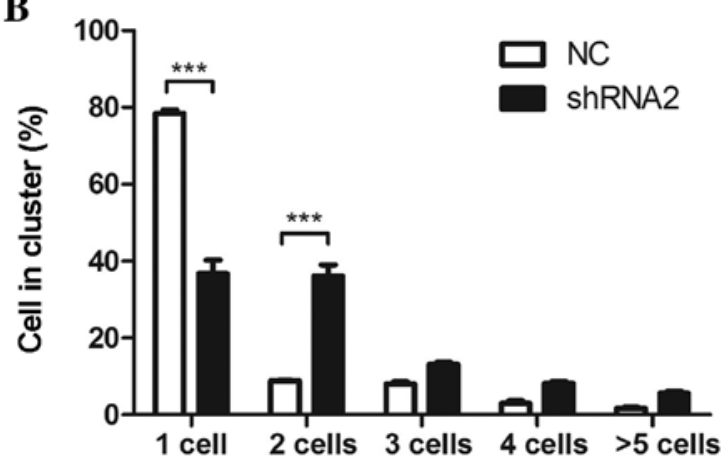

Figure 4. Knockdown of the erythropoietin-producing hepatocellular receptor B2 (EphB2) promotes cell-cell adhesion in U87 glioma cells (A) Two shRNAs, shRNA1 and shRNA2, were designed to knock down EphB2. Immunoblotting was used to measure endogenous EphB2 expression in U87 cells transfected with shRNA1, shRNA2 or a shRNA-control [negative control (NC)] expression vector. $\beta$-actin was used as a loading control and normalized band densities are shown in the figure. (B) Quantification of U87 cell clustering in EphB2 shRNA2 expressing cells. Cells were plated at moderate density, cultured for $48 \mathrm{~h}$, and the cell adhesion assay was performed. One hundred fields of view were randomly selected, and the percentage of U87 cells in clusters of increasing size was calculated. Values are mean \pm SD $(n=3)$ of one representative experiment from several replicates; ${ }^{* * * *} \mathrm{P}<0.001$.

miR-128 inhibits glioma cell migration via EphB2. To evaluate the effect of miR-128 on the regulation of EphB2 in glioma cell migration, a transwell assay and a wound-healing assay were performed. The overexpression of miR-128 decreased the cell migration using transwell assay as shown in Fig. 6A and D. An artificial wound was made $24 \mathrm{~h}$ after U87-miR-128, negative control and U87 cells were plated, both phase-contrast photomicrograph and fluorescent photomicrograph were captured. Migration into the wound was measured at 12 and $24 \mathrm{~h}$. Migration was significantly decreased in U87-miR-128 cells (Fig. 6B and E); however, the effect of miR-128 was inhibited in cells co-expressing EphB2 (Fig. 6C and F). These findings indicate that EphB2 overexpression antagonizes the effect of miR-128 inhibition on U87 cell migration. Collectively, these results provide evidence that miR-128 promotes cell-cell adhesion and suppresses cell migration in glioma cells in a mechanism dependent on EphB2 expression.

\section{Discussion}

Brain-enriched miR-128 has been shown to be downregulated in glioma tissues and cell lines (12) and it has also been shown to regulate cell death/survival and invasion by various types of cancer. miR-128 could target different genes in a certain tissue or cell lines and have different functions. Two important studies have shown that miR-128 can suppress glioma cell proliferation by targeting E2F3a and reduce glioma self-
A
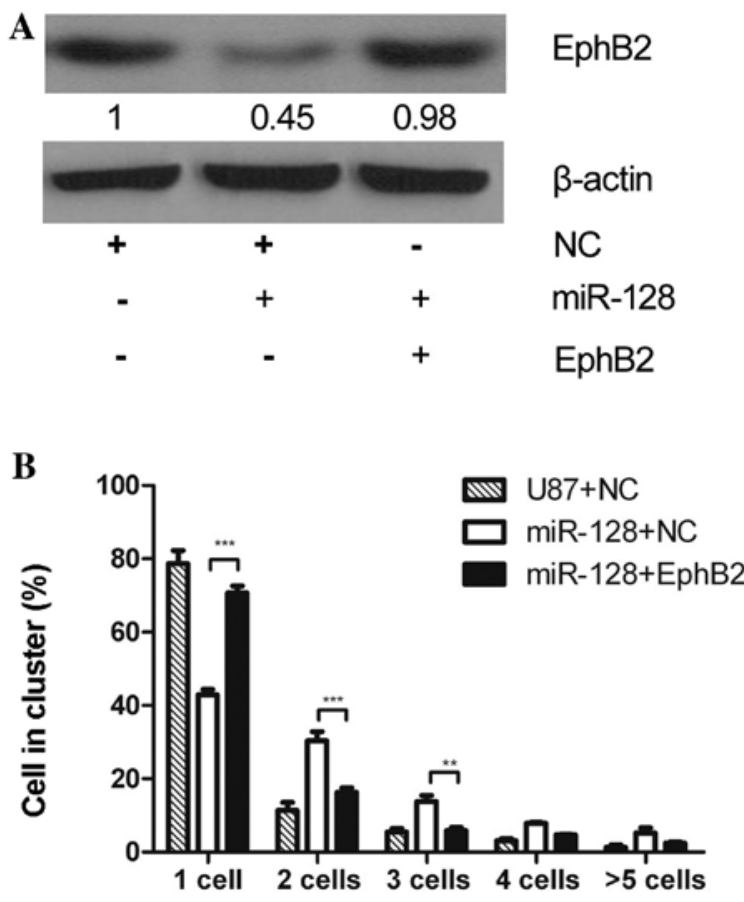

Figure 5. The erythropoietin-producing hepatocellular receptor B2 (EphB2) suppresses the effect of miR-128 on cell-cell adhesion. (A) Immunoblotting of EphB2 expression in U87 cells transfected with empty vector (U87 + NC) and in stably expressing miR-128 U87 cells (miR-128) transfected with EphB2 expression vector $(\mathrm{miR}-128+\mathrm{EphB} 2)$ or empty vector $(\mathrm{miR}-128+\mathrm{NC})$ $\beta$-actin was used as a loading control and normalized band densities were shown in the figure. (B) Quantification of cell clustering in U87 cells coexpressing miR-128 and/or EphB2. U87 cells were transfected with empty vector (U87 + NC) as control, stably expressing miR-128 U87 cells were transfected with an EphB2 expression vector (miR-128 + EphB2) or empty vector (miR-128 + NC), cultured for $48 \mathrm{~h}$, then the cell-cell adhesion assay was performed. A total of 100 fields of view were randomly selected, and the number of clusters containing different number of cells was determined. Values are mean $\pm \mathrm{SD}(\mathrm{n}=3)$ of one representative experiment from several replicates; ${ }^{* * *} \mathrm{P}<0.001,{ }^{* *} \mathrm{P}<0.01$

renewal by targeting Bmi-1 or RTK signaling $(12,13)$. miR-128 inhibits tumor growth and angiogenesis by targeting p70S6K1 (12). Additionally, miR-128 has been linked to neuroblastoma cell motility and invasiveness by targeting Reelin and DCX (14). In the present study, we reported for the first time that miR-128 promotes cell-cell adhesion and inhibits cell migration in glioma cells through a novel target EphB2, a gene relative to cell-cell adhesion. Overexpression of EphB2 could rescue the function of miR-128.

miRNAs play a critical role in gene regulation; however, the regulation of Eph family members by miRNAs is poorly characterized. Recently, miR-26b was found to directly regulate EphA2 expression via a specific binding site in the 3'UTR region of EphA2 mRNA (25). To our knowledge, brain-enriched miR-128 is the second miRNA found to regulate Eph family members. Our study indicates that EphB1 and EphB2 are novel targets of miR-128, and several lines of evidence support a direct interaction between miR-128 and the EphB1 and EphB2 3'UTRs. Firstly, the human EphB1 and EphB2 3'UTRs both contain a putative miR-128 binding site with a prominent seed match (Fig. 2B). Secondly, miR-128 suppresses the activity of luciferase reporter genes fused to the 3'UTR of either EphB1 or EphB2 mRNA. Thirdly, miR-128 


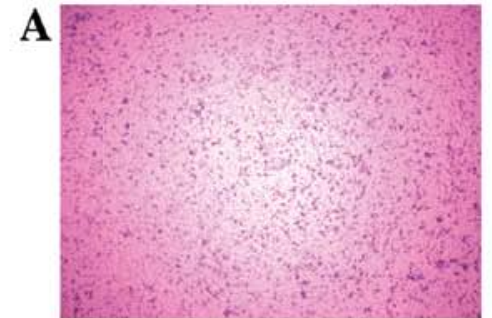

U87

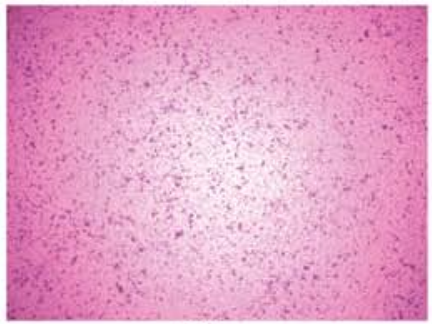

NC

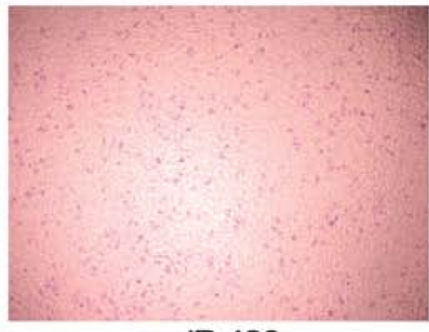

miR-128
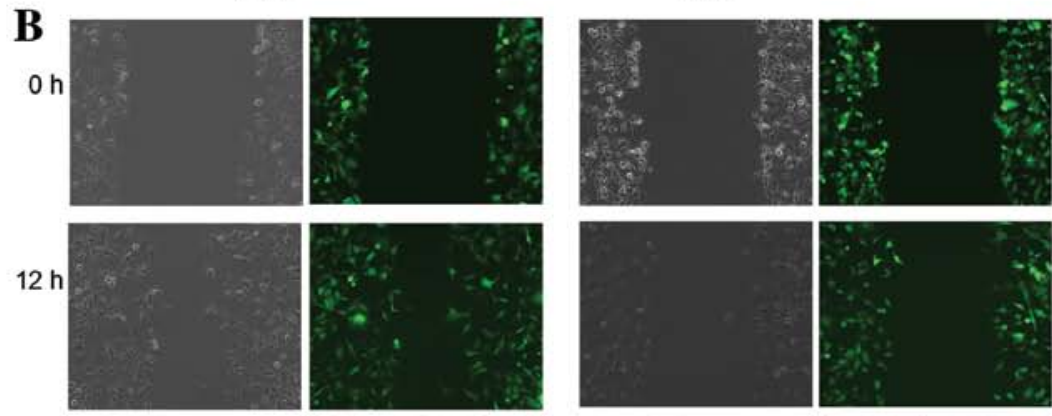

$24 \mathrm{~h}$
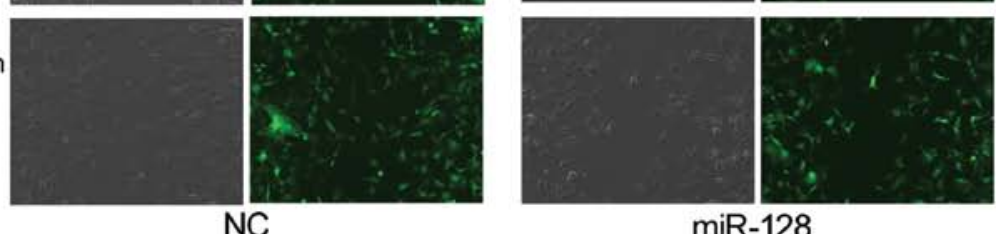

$\operatorname{miR}-128$
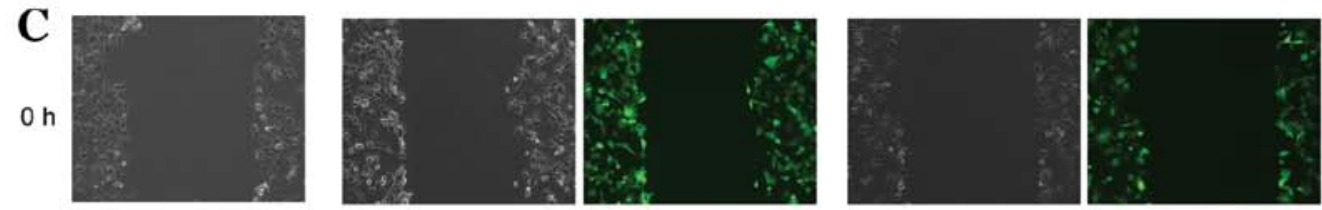

$12 \mathrm{~h}$
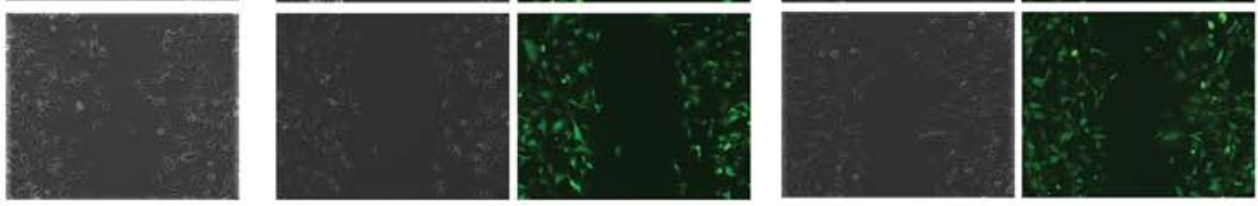

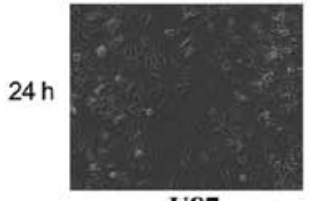

U87

D

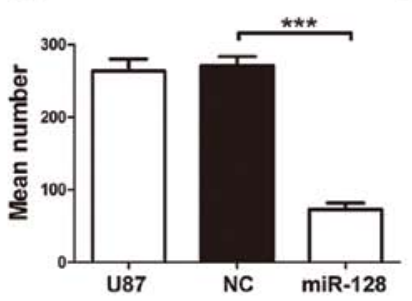

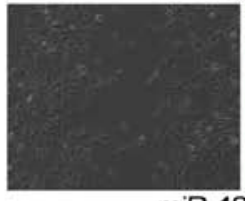

miR-128+NC

$\mathbf{E}$
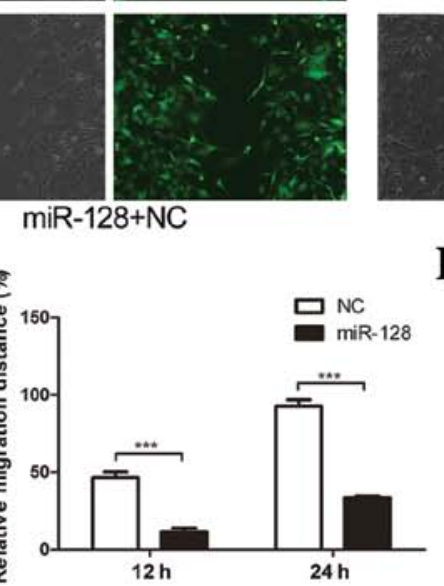
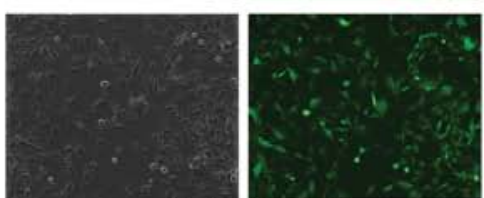

miR-128+EphB2

$\mathbf{F}$

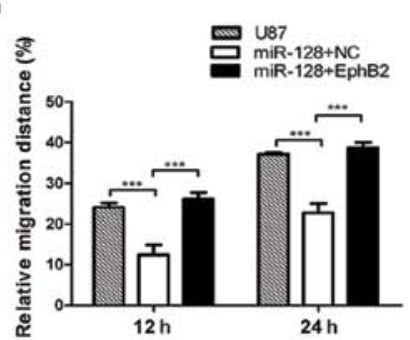

Figure 6. miR-128 affects glioma cell migration. (A) The migration assay was set up in transwell chambers. Cell culture inserts with 8.0 -mm pore diameter were used to separate the top and bottom chambers. U87 cells, U87 cells infected with empty vector lentivirus [negative control (NC)], stable miR-128-expressing U87 cells (miR-128) were plated onto the top chamber. After $4 \mathrm{~h}$, the cells had invaded to the lower surface of the membrane. Images show cells migrated to the bottom of chambers on the inserts. (B) The wound-healing assay was employed to investigate the effect of miR-128 on glioma cell migration. Stable miR-128-expressing U87 cells (miR-128) were cultured until 90\% confluent and an artificial, homogenous wound was then created. U87 cells infected with empty vector lentivirus served as an NC. (C) Wound-healing assay was employed to investigate whether the erythropoietin-producing hepatocellular receptor B2 (EphB2) could rescue the suppressive effect of miR-128 on glioma cell migration. Stable miR-128-expressing U87 cells (miR-128) were transfected with the EphB2 expression vector pEphB2 (miR-128 + EphB2) or empty vector (miR-128 + NC), and an artificial, homogenous wound was created. Representative images were captured at 0,12 and $24 \mathrm{~h}$ post-wounding with magnification, $\mathrm{x} 100$ and from left to right are images of bright field and fluorescent photomicrograph in (A and B). (D) miR-128 markedly reduced U87 cell migration in vitro. Quantification of U87 glioma cells as in (A). Data are presented as mean \pm SD for the experiment. ${ }^{* * *} \mathrm{P}<0.001$, statistically significant difference. (E) Quantification of U87 glioma cell migration in miR-128 and NC cells in (B). (F) Quantification of U87 glioma cell migration in miR-128 cells transfected with EphB2 or NC. The gap remaining between the migrating cells is expressed as a percentage of the initial wound width $\left({ }^{* * *} \mathrm{P}<0.001\right)$. 
suppresses endogenous expression of human EphB2 at the protein level. We observed that cell-cell adhesion increased and cell migration decreased when miR-128 was overexpressed in U87 glioma cells, which suggests that miR-128 affects cell-cell adhesion and cell migration in glioma cells via downregulation of EphB2. This hypothesis was confirmed by the rescue experiments. Overexpression of EphB2 without the 3'UTR, which the miR-128 has no effect on, inhibited the ability of miR-128 to promote cell-cell adhesion and suppress cell migration in U87 cells. This study provides the first identification and demonstration of a miRNA which directly regulates EphB1 and EphB2 and this, in turn, enhances our understanding of the function and regulation of EphB2 in glioma cells.

Several Ephs and ephrin ligands are expressed at high levels in multiple types of cancer where they demonstrate mostly tumor promoting activities (reviewed in ref. 22). High expression levels of EphB2 have been reported in a variety of tumors such as gliomas $(16,17)$, synovial sarcomas (26), liver cancer (27), gastric cancer (28), colon carcinomas (29), lung cancer (30) and breast cancer (31). In human brain tumors, EphB2 expression negatively correlates with tumor grade (16). EphB2 is involved in malignant progression in glioma tissues, as more migratory glioma cells express higher levels of EphB2, and ectopic overexpression of EphB2 promotes glioma cell migration and invasion. In agreement with these observations, blocking EphB2 expression in glioma cells significantly inhibits migration and invasion (16). The molecular mechanisms which regulate the high levels of EphB2 expression in glioma tissues and cell lines are not known. In this study, we demonstrated that miR-128 binds the 3'UTR of EphB2 mRNA to post-transcriptionally regulate EphB2 expression. miR-128 is downregulated in gliomas $(6,9)$, which, in turn, could lead to increased expression levels of EphB2. Furthermore, our observation that miR-128-induced downregulation of EphB2 inhibits glioma cell migration is consistent with previous studies which have reported that low expression levels of EphB2 can block a variety of malignant processes in tumor cells $(16,17)$.

EphB2 mediates repulsion and adhesion of nerve cells $(15,21,23)$ and once cell-cell contact occurs, separation of interacting cells is difficult due to high expression of EphB receptors on the cell surface and the high affinity of EphBEphrinB binding. To separate two interacting cells, the connection between EphB and EphrinB at contact sites has to be destroyed, and two mechanisms have been proposed: clearing of the receptor or ligand by a transmembrane protease or removal of the EphB-EphrinB complex interaction by endocytosis and trans-endocytosis (22). Increased expression of EphB receptors can reduce cell-cell contact and promote cell-cell repulsion via endocytosis of activated ephrinB-EphB receptors, leading to a reduced number of contact sites between receptor-expressing and ligandexpressing cells (32). As regards U87 glioma cells, we propose that high levels of EphB2 expression are mediated by miR-128 downregulation, leading to increased endocytosis of the EphB-EphrinB complex, which reduces cell-cell adhesion and promotes glioma cell migration. Conversely, overexpression of miR-128 reduces EphB2 expression and promotes cell-cell adhesion.
In summary, our findings suggest that the aberrant expression of miR-128 in gliomas plays a critical role in the regulation of cell-cell adhesion and cell migration. The function of miR-128 in cell-cell adhesion and cell migration is dependent on regulation of the EphB2 receptor by direct targeting of EphB2 mRNA via binding the 3'UTR. The present study provides evidence for a novel function of miR-128 in the regulation of EphB2 in glioma cells.

\section{Acknowledgements}

This study was supported by grants from the National Natural Science Foundation of China (grant no. 81272773 and 81101960); the Scientific and Technological Planning of Guangzhou (grant no. 2012J4100082); the Fundamental Research Funds for the Central Universities (grant no. 10lgpy23).

\section{References}

1. Surawicz TS, Davis F, Freels S, Laws ER Jr and Menck HR: Brain tumor survival: results from the National Cancer Data Base. J Neurooncol 40: 151-160, 1998.

2. Bartel DP: MicroRNAs: genomics, biogenesis, mechanism, and function. Cell 116: 281-297, 2004.

3. Calin GA, Sevignani C, Dumitru CD, et al: Human microRNA genes are frequently located at fragile sites and genomic regions involved in cancers. Proc Natl Acad Sci USA 101: 2999-3004, 2004.

4. Chen CZ: MicroRNAs as oncogenes and tumor suppressors. N Engl J Med 353: 1768-1771, 2005.

5. Croce CM and Calin GA: miRNAs, cancer, and stem cell division. Cell 122: 6-7, 2005.

6. Ciafre SA, Galardi S, Mangiola A, et al: Extensive modulation of a set of microRNAs in primary glioblastoma. Biochem Biophys Res Commun 334: 1351-1358, 2005.

7. Silber J, Lim DA, Petritsch C, et al: miR-124 and miR-137 inhibit proliferation of glioblastoma multiforme cells and induce differentiation of brain tumor stem cells. BMC Med 6: $14,2008$.

8. Monteys AM, Spengler RM, Wan J, et al: Structure and activity of putative intronic miRNA promoters. RNA 16: 495-505, 2010.

9. Lages E, Guttin A, El Atifi M, et al: MicroRNA and target protein patterns reveal physiopathological features of glioma subtypes. PLoS One 6: e20600, 2011.

10. Guidi M, Muinos-Gimeno M, Kagerbauer B, Marti E, Estivill $\mathrm{X}$ and Espinosa-Parrilla $\mathrm{Y}$ : Overexpression of miR-128 specifically inhibits the truncated isoform of NTRK3 and upregulates BCL2 in SH-SY5Y neuroblastoma cells. BMC Mol Biol 11: 95, 2010.

11. Adlakha YK and Saini N: MicroRNA-128 downregulates Bax and induces apoptosis in human embryonic kidney cells. Cell Mol Life Sci 68: 1415-1428, 2011.

12. Zhang Y, Chao T, Li R, et al: MicroRNA-128 inhibits glioma cells proliferation by targeting transcription factor E2F3a. J Mol Med 87: 43-51, 2009.

13. Godlewski J, Nowicki MO, Bronisz A, et al: Targeting of the Bmi-1 oncogene/stem cell renewal factor by microRNA-128 inhibits glioma proliferation and self-renewal. Cancer Res 68: 9125-9130, 2008.

14. Evangelisti C, Florian MC, Massimi I, et al: MiR-128 up-regulation inhibits Reelin and DCX expression and reduces neuroblastoma cell motility and invasiveness. FASEB J 23: 4276-4287, 2009.

15. Halloran MC and Wolman MA: Repulsion or adhesion: receptors make the call. Curr Opin Cell Biol 18: 533-540, 2006.

16. Nakada M, Niska JA, Miyamori H, et al: The phosphorylation of EphB2 receptor regulates migration and invasion of human glioma cells. Cancer Res 64: 3179-3185, 2004.

17. Nakada M, Niska JA, Tran NL, McDonough WS and Berens ME: EphB2/R-Ras signaling regulates glioma cell adhesion, growth, and invasion. Am J Pathol 167: 565-576, 2005.

18. Shen X, Fang J, Lv X, et al: Heparin impairs angiogenesis through inhibition of microRNA-10b. J Biol Chem 286: 26616-26627, 2011. 
19. Smirnova L, Grafe A, Seiler A, Schumacher S, Nitsch R and Wulczyn FG: Regulation of miRNA expression during neural cell specification. Eur J Neurosci 21: 1469-1477, 2005.

20. John B, Enright AJ, Aravin A, Tuschl T, Sander C and Marks DS Human MicroRNA targets. PLoS Biol 2: e363, 2004.

21. Kullander K and Klein R: Mechanisms and functions of Eph and ephrin signalling. Nat Rev Mol Cell Biol 3: 475-486, 2002.

22. Himanen JP, Saha N and Nikolov DB: Cell-cell signaling via Eph receptors and ephrins. Curr Opin Cell Biol 19: 534-542, 2007.

23. Flanagan JG and Vanderhaeghen P: The ephrins and Eph receptors in neural development. Annu Rev Neurosci 21: 309-345, 1998.

24. Bartel DP: MicroRNAs: target recognition and regulatory functions. Cell 136: 215-233, 2009.

25. Wu N, Zhao X, Liu M, et al: Role of microRNA-26b in glioma development and its mediated regulation on EphA2. PLoS One 6: e16264, 2011.

26. Barco R, Hunt LB, Frump AL, et al: The synovial sarcoma SYT-SSX2 oncogene remodels the cytoskeleton through activation of the ephrin pathway. Mol Biol Cell 18: 4003-4012, 2007.
27. Hafner C, Schmitz G, Meyer S, et al: Differential gene expression of Eph receptors and ephrins in benign human tissues and cancers. Clin Chem 50: 490-499, 2004.

28. Kataoka H, Tanaka M, Kanamori M, et al: Expression profile of EFNB1, EFNB2, two ligands of EPHB2 in human gastric cancer. J Cancer Res Clin Oncol 128: 343-348, 2002.

29. Liu W, Ahmad SA, Jung YD, et al: Coexpression of ephrin-Bs and their receptors in colon carcinoma. Cancer 94: 934-939, 2002.

30. Tang XX, Brodeur GM, Campling BG and Ikegaki N: Coexpression of transcripts encoding EPHB receptor protein tyrosine kinases and their ephrin-B ligands in human small cell lung carcinoma. Clin Cancer Res 5: 455-460, 1999.

31. Wu Q, Suo Z, Risberg B, Karlsson MG, Villman K and Nesland JM: Expression of Ephb2 and Ephb4 in breast carcinoma. Pathol Oncol Res 10: 26-33, 2004.

32. Marston DJ, Dickinson S and Nobes CD: Rac-dependent transendocytosis of ephrinBs regulates Eph-ephrin contact repulsion. Nat Cell Biol 5: 879-888, 2003. 